\title{
Commentary: "An Imaging Overview of the Posterior Septum of the Knee and Trans-Septal Portal Procedure: Normal Anatomy, Indications, and Unique Imaging Considerations"
}

\author{
Jay Moran', Mark D. Miller², Michael J. Medvecky ${ }^{1 *}$
}

'Department of Orthopaedics and Rehabilitation, Yale School of Medicine, New Haven, CT, USA

${ }^{2}$ Department of Orthopaedics, University of Virginia, Charlottesville, VA, USA

\section{Article Info}

\section{Article Notes}

Received: August 03, 2021

Accepted: September 07, 2021

\section{*Correspondence:}

*Dr. Michael J. Medvecky, Vice-Chair for Faculty Affairs,

Associate Professor, Section of Sports Medicine; Department of Orthopaedics and Rehabilitation, Yale School of Medicine, New Haven, CT, USA; Telephone No: 203-785-6907; Email: Michael.Medvecky@yale.edu.

${ }^{\circledR} 2021$ Medvecky MJ. This article is distributed under the terms of the Creative Commons Attribution 4.0 International License.

\section{Introduction}

Knee arthroscopy has allowed surgeons to treat a variety of pathologies with minimally invasive maneuvers compared to traditional open approaches ${ }^{1-4}$. However, access to the posterior compartment remains a challenge with traditional anterior portals in arthroscopic knee procedures ${ }^{5,6}$. The posterior trans-septal portal (TSP) technique involves the creation of a connecting accessory pathway through the posterior septum of the knee that separates the posteromedial and posterolateral compartments ${ }^{4,6}$. This portal allows surgeons to pass surgical instruments back and forth across these compartments and can provide a wider visualization of the posterior anatomy compared to traditional approaches ${ }^{7}$. However, there is limited literature that has described the technique, despite its elimination of blind spots when viewing and treating posterior pathologies of the knee. The main purpose of our article, "An Imaging Overview of the Posterior Septum of the Knee and TransSeptal Portal Procedure: Normal Anatomy, Indications, and Unique Imaging Considerations" was to provide a thorough radiological review of the TSP technique with an emphasis on the indications, surrounding posterior anatomy, and imaging considerations ${ }^{8}$. In this commentary, we provide a more general overview of the TSP technique with references to this article and the current literature. By doing so, we hope to spread awareness of this underutilized technique and provide the groundwork for future research.

\section{Trans-Septal Portal Technique}

The first step in the procedure involves proper patient positioning with the knee flexed to $90^{\circ}$ which creates the furthest distance from the surrounding neurovascular structures. Standard anterior portals are then made and diagnostic arthroscopy is carried out. A posteromedial portal is created by advancing the arthroscope from the anterolateral portal into the posteromedial compartment. A spinal needle with transillumination can be used for proper placement of the posteromedial portal. The posterolateral portal can be made in a similar fashion as the posteromedial portal using a trans-notch view from the anteromedial portal or by passing the arthroscope over the posterior horn of the lateral meniscus to a position overlying the posterior root of the lateral meniscus, which will then allow visual access to the posterolateral joint capsule. Once the posterolateral portal is created, the arthroscope can then be positioned in the posteromedial portal to view the posterior 
septum. Perforating the posterior septum from a lateral to medial direction decreases the risk of iatrogenic injury to the popliteal artery, which is situated closer to the posterolateral aspect of the joint capsule at this level. Creation of the TSP from the opposite direction could allow for the instrument to pass out the posterolateral joint capsule into the direction of the popliteal artery ${ }^{7}$. A blunt trocar or switching stick passed through the posterolateral portal can be used to determine the position when perforating the posterior septum which can also be confirmed by placing a long spinal needle then through the septum. After the spinal needle has penetrated the posterior septum, with adequate visualization from the posteromedial compartment, a blunt instrument can be passed through the septum to create the TSP. The TSP can now be opened up by placing a shaver into this space and debriding the posterior septum. Working instruments can now be passed back and forth from compartment to compartment $t^{4,6,9,10}$. Please refer to Figure 1 and Figure 8 in our original article which shows a diagrammatic representation of TSP portal creation and posterolateral \& posteromedial portal creation, respectively.

\section{Indications}

As indicated in our original article, the increased visualization of the posterior knee from the TSP can facilitate technically difficult procedures that are encountered with the anterior arthroscopic approach, as reports have shown that there is a blind zone of $8.4 \%$, on average, between the anterior and posterior visual field ${ }^{4}$. With the TSP technique, this blind zone is eliminated and can greatly aid in removal of posterior lesions, such as tumors or loose bodies that need to be resected entirely ${ }^{4,6}$. Moreover, recent reports have used the TSP technique for treatment of ramp lesions, or tears to the posterior horn of the medial meniscus, which are difficult to detect and treat using standardized anterior portals ${ }^{11}$. Within the literature, Buyukdogan et al. showed that these pathologies can be successfully visualized and treated using the TSP technique, with excellent visualization of the lesion ${ }^{3}$. Additionally, reports have shown that the TSP can be utilized for posterior cruciate ligament (PCL) reconstructions and PCL tibial avulsion fracture fixations ${ }^{4,6,12}$. Especially during PCL reconstructions, the passage of the graft during the "killer turn" can be more easily facilitated with posterior and trans-septal portals ${ }^{2}$. Incorrect placement of the graft has been associated with poorer outcomes in PCL reconstructions ${ }^{2}$. Other indications for using the TSP include posterior cyst removal, pigmented villonodular synovitis, and posterior capsular release for extension loss ${ }^{4,6,12}$.

Please refer to Figures 5, 6 and 9 in the original article for more in-depth indications and case examples.

\section{Anatomical Review}

The imaging considerations described in our article focus on the main neurovascular structures surrounding the three posterior portals utilized in this technique. Preoperative imaging, which typically involves a standard knee MRI, can help detect any neurovascular variants in the structures that lie in close proximity to the portals. In our original article, we introduce a flexed knee MRI, which provide a more thorough understanding of the anatomical features surrounding the TSP in the position of the knee during surgery. While a flexed knee MRI is not necessary for this procedure, it may be useful for surgical planning if a neurovascular variation is detected on standard MRI.

When creating the posteromedial, posterolateral portal, and trans-septal portal, the knee should be flexed 90 degrees to provide the furthest distance from the saphenous vein $(16-28 \mathrm{~mm})$ on the medial side, the peroneal nerve $(32-40 \mathrm{~mm})$ on the lateral side, and the popliteal artery near the posterior capsule and septum ${ }^{13-17}$. Approximately, $90^{\circ}$ of knee flexion provides the greatest distance from the respective anatomical structure(s) and should be maintained throughout the procedure. In our original article, please refer to Figures 2, 7, and 10 for an MRI anatomical review surrounding the TSP.

\section{Future Outlook}

Despite its creation in 1997, wide-spread adoption seems to be lacking given the limited literature on the technique. To our knowledge, the literature has reported over 700 knee surgeries over a 20-year period using the trans-septal portal with no reports of iatrogenic neurovascular injury ${ }^{4,6,9,12}$. However, these reports come from small cohort studies and technical articles which warrants future randomized control studies to assess the true incidence of complications.

This surgical procedure is technically challenging, even for well-trained surgeons due to the surrounding neurovascular structures that are not typically encountered with traditional anterior approaches. Sports medicine fellowships may benefit from integrating this technique into their program, if they have not already. For surgeons comfortable with this technique, it offers many of the aforementioned advantages compared to the traditional anterior-posterior approach. Randomized control trials should be considered to compare the TSP technique to other commonly used posterior approaches for treating particular pathologies.

\section{Conclusions}

The posterior trans-septal portal technique allows for significantly enhanced visualization of the posterior compartment of the knee in a less-invasive way compared to open surgery. This technique has several advantages over 
the traditional anterior portal arthroscopic approaches to the knee. As noted in our article, thorough knowledge of the posterior anatomy, as well as the surrounding neurovascular structures, will benefit the surgeon looking to incorporate this technique into their surgical repertoire. Pre-operative imaging can help with the anatomical understanding of the posterior anatomy but is an understudied area regarding the TSP technique. More data on this procedure is needed as the current literature only consists of small cohort studies and technical reports. Such data would provide more insight into how this technique compares to other commonly used techniques and the importance of pre-operative planning.

\section{Disclosures}

The authors have no relevant conflict of interests with the current work.

\section{Funding}

No funding was used for this study.

\section{References}

1. Ahn JH, Wang JH, Yoo JC, et al. A pull out suture for transection of the posterior horn of the medial meniscus: Using a posterior trans-septal portal. Knee Surgery, Sport Traumatol Arthrosc. 2007; 15(12): 15101513. doi:10.1007/s00167-007-0310-3

2. Ahn JH, Chung YS, Oh I. Arthroscopic posterior cruciate ligament reconstruction using the posterior trans-septal portal. Arthrosc - I Arthrosc RelatSurg. 2003; 19(1): 101-107. doi:10.1053/jars.2003.50017

3. Buyukdogan K, Laidlaw MS, Miller MD. Meniscal Ramp Lesion Repair by a Trans-septal Portal Technique. Arthrosc Tech. 2017; 6(4): e1379-e1386. doi:10.1016/j.eats.2017.05.029

4. Ahn JH, Ha CW. Posterior trans-septal portal for arthroscopic surgery of the knee joint. Arthroscopy. 2000; 16(7): 774-779. doi:10.1053/ jars.2000.7681

5. Kramer DE, Bahk MS, Cascio BM, et al. Posterior knee arthroscopy: Anatomy, technique, application. J Bone Joint Surg Am. 2006; 88 Suppl 4:110-121. doi:10.2106/JBJS.F.00607

6. Ohishi $\mathrm{T}$, Takahashi M, Suzuki D, et al. Arthroscopic approach to the posterior compartment of the knee using a posterior transseptal portal. World J Orthop. 2015; 6(7): 505-512. doi:10.5312/wjo.v6.i7.505
7. Ahn JH, Lee SH, Jung HJ, et al. The relationship of neural structures to arthroscopic posterior portals according to knee positioning. Knee Surgery, Sport Traumatol Arthrosc. 2011; 19(4): 646-652. doi:10.1007/s00167-010-1326-7

8. Moran J, Porrino J, Cheng R, et al. An Imaging Overview of the Posterior Septum of the Knee and Trans-Septal Portal Procedure: Normal Anatomy, Indications, and Unique Imaging Considerations. Curr Probl Diagn Radiol. 2021; S0363-0188. doi:10.1067/j. cpradiol.2021.04.001

9. Louisia S, Charrois O, Beaufils P. Posterior "back and forth" approach in arthroscopic surgery on the posterior knee compartments. Arthrosc - J Arthrosc Relat Surg. 2003; 19(3): 321-325. doi:10.1053/ jars.2003.50082

10. Kim SJ, Song HT, Moon HK, et al. The safe establishment of a transseptal portal in the posterior knee. Knee Surgery, Sport Traumatol Arthrosc. 2011; 19(8): 1320-1325. doi:10.1007/s00167-011-1429-9

11. Arner JW, Herbst E, Burnham JM, et al. MRI can accurately detect meniscal ramp lesions of the knee. Knee Surg Sports Traumatol Arthrosc. 2017; 25(12): 3955-3960. doi:10.1007/S00167-017-45239

12. Kim JM. Direct posterior-posterior triangulation of the knee joint. Arthroscopy. 1997; 13(2): 262-264. doi:10.1016/S07498063(97)90167-5

13. Pace JL, Wahl CJ. Arthroscopy of the Posterior Knee Compartments: Neurovascular Anatomic Relationships During Arthroscopic Transverse Capsulotomy. Arthrosc - J Arthrosc Relat Surg. 2010; 26(5): 637-642. doi:10.1016/j.arthro.2009.09.009

14. Kramer DE, Bahk MS, Totty WG, et al. Proximity of the Popliteal Artery to the PCL During Simulated Knee Arthroscopy. J Knee Surg. 2006; 19(3): 181-185. doi:10.1055/s-0030-1248103

15. Jin HA, Joon HW, Sang HL, et al. Increasing the distance between the posterior cruciate ligament and the popliteal neurovascular bundle by a limited posterior capsular release during arthroscopic transtibial posterior cruciate ligament reconstruction: A cadaveric angiographic study. Am J Sports Med. 2007; 35(5): 787-792. doi:10.1177/0363546506297908

16. Makridis KG, Wajsfisz A, Agrawal N, et al. Neurovascular anatomic relationships to arthroscopic posterior and transseptal portals in different knee positions. Am J Sports Med. 2013; 41(7): 1559-1564. doi:10.1177/0363546513492704

17. Cancienne JM, Werner BC, Burrus MT, et al. The Transseptal Arthroscopic Knee Portal Is in Close Proximity to the Popliteal Artery: A Cadaveric Study. J Knee Surg. 2017; 30(9): 920-924. doi:10.1055/s-0037-1599252 